\title{
Multi-time scale coordinated control method for distribution network based on point of common coupling reactive power/voltage optimization of photovoltaic
}

\author{
Si-Liang Liu *, Wei Huang, Yong-Jun Zhang \\ School of Electrical Power, South China University of Technology, \\ No. 381, Wushan Road, Tianhe District, Guangzhou 510640, China
}

\begin{abstract}
To solve the voltage control problem caused by high penetration level of distributed photovoltaic (DPV) in distribution network (DN), based on the voltage control characteristics of the reactive power compensated PV and the voltage-controlled PV, a multi-time scale coordinated control method based on point of common coupling (PCC) reactive power/voltage optimization of PV is proposed. On the long-time scale, an optimal reactive power dispatch (ORPD) model considering the chance constraint of voltage is proposed, which sets minimum operating cost as its target, including the adjusting cost of discrete devices. On the short-time scale, an adaptively slack optimal control strategy for PCC reactive power and voltage (Q/V-ASOC) is proposed, which can adaptively adjust the PCC reactive power and voltage according to the real-time changes of PV and load output. By comparing the control effect of different coordinated control methods for multiple PVs, an advise on the decision of reactive power and voltage coordinated control scheme for DN with high penetration level of PV can be provided.
\end{abstract}

Keywords: distributed photovoltaic, point of common coupling reactive power/voltage, multi-time scale, chance constraint, coordinated control

\section{Introduction}

On account of the advantage of low cost, easy installation, flexible control, and environmental compatibility, distributed generation (DG) has been developed rapidly in recent years, especially DPV. However, large scale integration of DPV poses a huge challenge to the operation of DN [1]. At present, most of the research results show that the operation of DG in DN should take into account the centralized optimal dispatch on the long-time scale and the decentralized autonomous control on short-time scale. Due to the poor communication level of DN at this stage, it is difficult for the centralized optimal dispatch to issue real-time control commands and response to changing operation conditions [2]. The control effect of the decentralized autonomous control depends on the setting value of the control parameters, so the economic effect can not be guaranteed [3]. Therefore, most of the researchers take great interest in how to form a multi-time scale coordinated control method for DN with DG.

References [4] proposed an active and reactive power coordinated optimal dispatch for active distribution network (ADN) based on model predictive control to handle uncertainty of renewable energy and load, which is formed by day-ahead scheduling, intra-day rolling scheduling and real-time feedback correction. References [5] proposed an optimal coordination strategy considering multiple time-scales, which is based on an online moving horizon optimization approach. Reference [6] proposed a multi-time scale coordinated scheduling model with distributed power flow controller to minimize wind power spillage, including day-ahead and real-time scale model. Reference [7] firstly proposed an indicator of

\footnotetext{
* Manuscript received February 15, 2018; revised August 20, 2018.

Corresponding author. Tel.: +86-13660546485; E-mail address:463695442@qq.com.

doi: $10.12720 /$ sgce.8.5.580-585
} 
feeder control error (FCE) to represent differences between actual operating status and global optimized status, then build a constant interchange control and object tracking control model based on FCE for local autonomy control based on global optimization. Although the method mentioned above can meet the regulation requirements in DN with DG at different time scales, there are still two problems as follows: (1) The centralized optimal dispatch does not consider the node voltage chance constraint, which can easily lead to high risk of voltage violation and need to substantially adjust in real-time; (2) The decentralized autonomous control generally tracks a constant interchange power optimization value of DG or regional connection point, but such kind of constant power control method can easily cause the voltage fluctuation problem when the penetration of DG is higher.

Motivated by the observations above, based on the voltage control characteristics of reactive power compensated PV (Q-PV for short) and voltage-controlled PV (V-PV for short), this paper proposes a multi-time scale coordinated control method for DN based on PCC reactive power/voltage optimization of PV, including an ORPD model on the long-time scale and a Q/V-ASOC strategy on the short-time scale. Finally, the method is tested on modified IEEE33-bus test system. By comparing the control effect of Q-PV and V-PV accessed in different locations in DN, an advise on the decision of reactive power and voltage coordinated control scheme for DN with high penetration level of PV can be provided.

\section{ORPD Model on Long-time Scale}

In the ORPD model, the forecast error of PV and load is described by a normal distribution [1] and a chance constraint of voltage is built to avoid high probability of voltage violation. Besides, it should be emphasized that the ORPD model excludes the constraint of inverter capacity. The reasons are as follows: (1) The forecast error model is hard to reflect the real-time changes in PV and load output. Without the constraint of inverter capacity, the dispatch instructions will lead the PCC reactive power/voltage to track the optimal value cooperating with the ASOC strategy, which can give full play to the remaining capacity of inverter; (2) The inverter capacity requirement can be calculated, which can provide an advise for inverter capacity configuration.

\subsection{ORPD model based on Q-PV on long-time scale}

In the ORPD model, the forecast error of PV and load is described by a normal distribution [1] and a chance constraint of voltage is built to avoid high probability of voltage violation. Besides, it should be emphasized that the ORPD model excludes the constraint of inverter capacity. The reasons are as follows: (1) The forecast error model is hard to reflect the real-time changes in PV and load output. Without the constraint of inverter capacity, the dispatch instructions will lead the PCC reactive power/voltage to track the optimal value cooperating with the ASOC strategy, which can give full play to the remaining capacity of inverter; (2) The inverter capacity requirement can be calculated, which can provide an advise for inverter capacity configuration.

$$
\begin{aligned}
& \min F=\sum_{t=1}^{T} \overline{P_{\text {loss }}^{t}}+P_{\mathrm{T}}^{t}+P_{\mathrm{C}}^{t} \\
& P_{\mathrm{T}}^{t}=\Delta P_{\mathrm{T}} \sum_{i \in \Omega_{\mathrm{T}}}\left|K_{\mathrm{T} i}^{t}-K_{\mathrm{T} i}^{t-1}\right|, \quad P_{\mathrm{C}}^{t}=\Delta P_{\mathrm{C}} \sum_{j \in \Omega_{\mathrm{C}}}\left|N_{\mathrm{C} j}^{t}-N_{\mathrm{C} j}^{t-1}\right|
\end{aligned}
$$

In (1), $\overline{P_{\text {loss }}^{t}}$ is the expect value of active power loss at time $t ; P_{\mathrm{T}}^{t}$ and $P_{\mathrm{C}}^{t}$ represent the adjustment cost of capacitor and OLTC at time $t$, respectively; $\Delta P_{\mathrm{T}}$ and $\Delta P_{\mathrm{C}}$ represent the unit adjustment cost of capacitor and OLTC, respectively; $K_{\mathrm{T} i}^{t}$ is the top position of OLTC at bus $i$ at time $t ; N_{\mathrm{C} j}^{t}$ is switching group number of capacity at bus $j$ at time $t ; \Omega_{\mathrm{T}}$ and $\Omega_{\mathrm{C}}$ represent the set of nodes of OLTC and capacity, respectively.

The equality constraints are the active power and reactive power flow equations [8].

The inequality constraints of control variables are given as following: 


$$
\begin{array}{ll}
K_{\mathrm{T} i \text { min }}^{t} \leq K_{\mathrm{T} i}^{t} \leq K_{\mathrm{T} i \text { max }}^{t}, & i \in \Omega_{\mathrm{T}} \\
N_{\mathrm{C} j \text { min }}^{t} \leq N_{\mathrm{C} j}^{t} \leq N_{\mathrm{C} j \text {.max }}^{t}, & j \in \Omega_{\mathrm{C}}
\end{array}
$$

In (2), $K_{\text {Timin }}^{t}$ and $K_{\text {Timax }}^{t}$ represent the lower and upper bound of $K_{\mathrm{T} i}^{t}$, respectively; $N_{\mathrm{C} j \text { min }}^{t}$ and $N_{\mathrm{C} j \text { max }}^{t}$ represent the lower and upper bound of $N_{\mathrm{c} \text {, }}^{t}$, respectively.

The chance constraint of voltage is given by

$$
\operatorname{Pr}\left\{V_{k \text { min }}^{t} \leq V_{k}^{t} \leq V_{k \cdot \max }^{t}\right\} \geq a_{\mathrm{v}}, \quad k \in \Omega
$$

In (3), $V_{k}^{t}$ is the voltage magnitude at bus $k$ at time $t ; V_{k \cdot \min }^{t}$ and $V_{k \text { max }}^{t}$ represent the lower and upper bound of $V_{k}^{t}$, respectively; $\alpha_{\mathrm{V}}$ is the confidence level of voltage; $\Omega$ is the set of nodes in DN.

\subsection{ORPD model based on $Q-P V$ on long-time scale}

The control variables of ORPD model based on V-PV are $N_{\mathrm{C}}, K_{\mathrm{T}}$ and PCC voltage of V-PV $V_{\mathrm{pv}} . V_{\mathrm{pv}}$ is a constant value at each dispatch interval. So the difference between the ORPD model based on V-PV and that based on Q-PV is that the former adds an inequality constraints of $V_{\mathrm{pv}}$, mathematically

$$
V_{\mathrm{pv} h \text { min }}^{t} \leq V_{\mathrm{pv} h}^{t} \leq V_{\mathrm{pv} h \text { max }}^{t}, \quad h \in \Omega_{\mathrm{V}-\mathrm{PV}}
$$

In (4), $V_{\mathrm{pv} h}^{t}$ is the PCC voltage magnitude of $\mathrm{V}-\mathrm{PV}$ at bus $h$ at time $t ; V_{\mathrm{pv} h . \text { min }}^{t}$ and $V_{\mathrm{pv} h . \max }^{t}$ represent the lower and upper bound of $V_{\mathrm{pv} h}^{t}$, respectively; $\Omega_{\mathrm{V}-\mathrm{PV}}$ is the set of nodes of V-PV.

According to the "three-sigma" rule of normal distribution, the inverter capacity requirement of Q-PV and V-PV can be calculated as following:

$$
S_{\mathrm{pvg} . \text { req }}^{(\mathrm{Q})}=\max _{t=1,2, \cdots, T}\left[\sqrt{\left(\mu_{\mathrm{Ppvg}}^{t}+3 \sigma_{\mathrm{Ppvg}}^{t}\right)^{2}+\left(Q_{\mathrm{pvg} . \mathrm{set}}^{t}\right)^{2}}\right], \quad g \in \Omega_{\mathrm{Q}-\mathrm{PV}}
$$

In (5), $S_{\mathrm{pvg} \text {.req }}^{(\mathrm{Q})}$ is the capacity requirement of Q-PV at bus $g$; $\mu_{\mathrm{P} \text { pvg }}^{t}$ and $\sigma_{\mathrm{P} p g}^{t}$ represent the expect value and standard deviation of predicted active power of Q-PV at bus $g$ at time $t$, respectively; $Q_{\text {pvgset }}^{t}$ is the scheduling value of PCC reactive power of Q-PV at bus $g$ at time $t ; \Omega_{\mathrm{Q}-\mathrm{PV}}$ is the set of nodes of Q-PV.

$$
S_{\mathrm{pv} h \text {.req }}^{(\mathrm{V})}=\max _{t=1,2, \cdots, T}\left[\sqrt{\left(\mu_{\mathrm{Ppv} h}^{t}+3 \sigma_{\mathrm{Ppv} h}^{t}\right)^{2}+\left(\mu_{\mathrm{Qpv} h}^{t}+3 \sigma_{\mathrm{Qpvh}}^{t}\right)^{2}}\right], \quad h \in \Omega_{\mathrm{V}-\mathrm{PV}}
$$

In (6), $S_{\mathrm{pv} h \text {.req }}^{\mathrm{V})}$ is the capacity requirement of V-PV at bus $h ; \mu_{\mathrm{Ppv} h}^{t}$ and $\sigma_{\mathrm{Ppv} h}^{t}$ represent the expect value and standard deviation of predicted active power of V-PV at bus $h$ at time $t$, respectively; $\mu_{\mathrm{Qpv} h}^{t}$ and $\sigma_{\mathrm{Qpv} h}^{t}$ represent the expect value and standard deviation of reactive power of V-PV at bus $h$ at time $t$.

A modified Cataclysmic GA embedded probabilistic method based on cumulant is introduced to solve the ORPD model [8], not to go into detail.

\section{PCC Q/V-ASOC Strategy on Short-time Scale}

Because the ORPD model excludes the constraint of inverter capacity and the forecast error can not fully cover all possibilities of PV and load output, the PCC reactive power/voltage may not track the scheduling value. So a Q/V-ASOC strategy is proposed.

\subsection{PCC Q-ASOC strategy for $Q-P V$ on short-time scale}

In the real-time control, if the reactive capacity is enough, then the PCC reactive power can be adjusted to track the scheduling value, otherwise it will be adjusted to the maximum value allowed and kept the state (output or absorb) the same to the scheduling value. The control block diagram of PCC QASOC strategy for Q-PV is shown in Fig. 1. (a).

In Fig. 1. (a), $Q_{\mathrm{pv} . \mathrm{set}}^{t}$ is the scheduling value of PCC reactive power of Q-PV at time $t ; P_{\mathrm{pv}}^{t}$ is the active power collection value of Q-PV at time $t ; Q_{\mathrm{pv} \text { min }}^{t}$ and $Q_{\mathrm{pv} \text { max }}^{t}$ represent the lower and upper bound of reactive 
power capacity of inverter at time $t$, respectively; $Q_{\mathrm{pv} \text {.mod }}^{t}$ is the modified reference value of reactive power of Q-PV at time $t ; Q_{\mathrm{PCC}}^{t}$ is the actual value of reactive power of Q-PV at time $t .1 /(T \mathrm{~s}+1)$ is the inertia element, which is equivalent to the inverter, and $T$ is the inertia time constant.

\subsection{PCC V-ASOC strategy for V-PV on short-time scale}

The principle of the PCC V-ASOC Strategy for V-PV is the same to that in Section 3.1.

The control block diagram is shown in Fig. 1. (b). $V_{\text {pv.set }}^{t}$ is the scheduling value of PCC voltage of V$\mathrm{PV}$ at time $t ; S_{\mathrm{VQ}}$ is the sensitivity parameter of PCC reactive power to voltage; $V_{\mathrm{PCC}}^{t}$ is the actual value of voltage of V-PV at time $t . f\left(P_{\mathrm{pv}}^{t}\right)$ is a disturbing variable which represent the impact of PCC active power to voltage; $k_{p}$ and $k_{i}$ represent the coefficients of the PI controller, respectively. The mathematical model of the comparing element in Fig. 1. (a) and Fig. 1. (b) are the same, and they can be expressed by

$$
\begin{cases}Q_{\mathrm{pv} . \bmod }^{t}=Q_{\mathrm{pv} . \text { set }}^{t}, & Q_{\mathrm{pv} . \min }^{t} \leq Q_{\mathrm{pv} . \text { set }}^{t} \leq Q_{\mathrm{pv} \text { max }}^{t} \\ Q_{\mathrm{pv} \text {.mod }}^{t}=Q_{\mathrm{pv} \text { max }}^{t}, & Q_{\mathrm{pv} . \text { set }}^{t}>Q_{\mathrm{pv} \text { max }}^{t} \\ Q_{\mathrm{pv} \text {.mod }}^{t}=Q_{\mathrm{pv} \text { min }}^{t}, & Q_{\mathrm{pv} \text {.set }}^{t}<Q_{\mathrm{pv} \text { min }}^{t}\end{cases}
$$

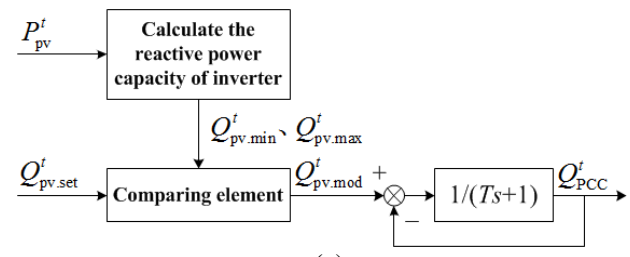

(a)

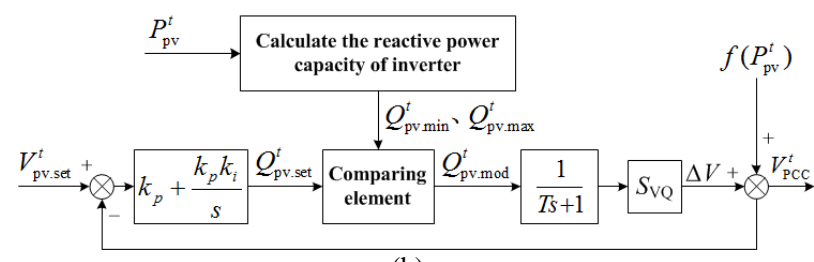

(b)

Fig. 1. The control block diagram of PCC Q/V-ASOC strategy: (a) for Q-PV and (b) for V-PV.

\section{Case Study}

This paper selects modified IEEE33-bus test system as the simulation example [8]. There are two PVs of the same capacity accessed dispersedly in the system. The location of them are arbitrarily chosen from three alternative nodes (node 3, node 10 and node 17), which represent the head, middle and tail of the feeder. Considering the types of PV, there are twelve juxtaposed scenarios. By solving the ORPD model under each scenario, the results are collected in Table 1.

Table 1. Control effect of each dispatching scheme

\begin{tabular}{cccccccc}
\hline Scenario & Scheme & $F / \mathrm{kW}$ & $\overline{P_{\text {loss }}} / \mathrm{kW}$ & $\begin{array}{c}\text { Action times } \\
\text { of OLTC }\end{array}$ & $\begin{array}{c}\text { Action times } \\
\text { of capacity }\end{array}$ & $S_{\text {pv.req }} / \mathrm{MVA}$ & $V_{\text {viol }} / \%$ \\
\hline 1 & VV-ht & 1433.75 & 1424.55 & 8 & 6 & $2.71 / 2.24$ & 0 \\
2 & QV-ht & 1433.75 & 1424.55 & 8 & 6 & $2.33 / 2.25$ & 0 \\
3 & QQ-ht & 1565.81 & 1552.21 & 10 & 18 & $3.33 / 2.22$ & 1 \\
4 & VQ-ht & 1558.47 & 1545.67 & 10 & 14 & $4.08 / 2.22$ & 0.99 \\
\hline 5 & VV-mt & 2411.16 & 2401.96 & 8 & 6 & $2.44 / 2.72$ & 1 \\
6 & QV-mt & 2419.93 & 2410.73 & 8 & 6 & $2.11 / 2.70$ & 1 \\
7 & QQ-mt & 2704.95 & 2694.55 & 8 & 12 & $2.30 / 2.71$ & 0.99 \\
8 & VQ-mt & 2528.00 & 2519.40 & 8 & 8 & $2.47 / 2.56$ & 1 \\
\hline 9 & VV-hm & 1043.13 & 1036.33 & 6 & 4 & $2.42 / 2.25$ & 0 \\
10 & QV-hm & 1043.13 & 1036.33 & 6 & 4 & $2.12 / 2.26$ & 0 \\
11 & QQ-hm & 1045.29 & 1038.49 & 6 & 4 & $2.08 / 2.08$ & 1 \\
12 & VQ-hm & 1044.04 & 1037.23 & 6 & 4 & $2.57 / 2.18$ & 1 \\
\hline \multicolumn{7}{r}{ For example, Scheme VV-ht means there are two V-PVs accessed in the head and tail of the feeder. }
\end{tabular}

According to Table 1, we can draw some conclusions as following:

(1) When two PVs access in the head and tail of the feeder, the result of Scheme VV-ht is the same to that of Scheme QV-ht except the inverter capacity requirement of PV, and they are better than the other two schemes obviously. Besides, comparing with Scheme QV-ht, the inverter capacity requirement of PV 
in the head of Scheme VV-ht is larger in order to keep the voltage constant and the growth reaches $15 \%$. So we advise to select Q-PV to access in the head and V-PV to access in the tail;

(2) When two PVs access in the middle and tail of the feeder, the result of Scheme VV-mt is close to that of Scheme QV-mt except the inverter capacity requirement of PV, and they are better than the other two schemes. Comparing with Scheme QV-mt, Scheme VV-mt gets better results in reducing power loss, but the inverter capacity requirement is larger; So we advise to select V-PV to access in the tail;

(3) When two PVs access in the head and middle of the feeder, the results of four schemes are almost the same on the aspect of power loss and action times of discrete devices. But the inverter capacity requirement of Scheme QQ-hm is the smallest, the inverter capacity of PV in the head of Scheme VV-hm and Scheme VQ-hm are much larger. So we advise to select Q-PV to access in the head.

Furthermore, this paper analyses the voltage fluctuation of each scheme by simulating the intra-day operation (1440 mins) under three kinds of inverter capacity set as 2.1 MVA (S1), 2.21 MVA (S2) and 2.31 MVA (S3), the maximum of voltage fluctuation $\Delta \mathrm{Vmax}$ can be gained as shown in Table 2 .

Table 2. The maximum of voltage fluctuation $\Delta V_{\max }$ under $S_{1} \sim S_{3}$ of each scheme

\begin{tabular}{cccc}
\hline \multirow{2}{*}{ Scheme } & \multicolumn{3}{c}{ Inverter capacity of PV } \\
\cline { 2 - 4 } & $S_{1}$ & $S_{2}$ & $S_{3}$ \\
\hline VV-ht & 0.0259 & 0.0068 & 0.0066 \\
QV-ht & 0.0273 & 0.0073 & 0.0071 \\
QQ-ht & 0.0462 & 0.0462 & 0.0462 \\
VQ-ht & 0.0431 & 0.0431 & 0.0431 \\
\hline VV-mt & 0.0423 & 0.0297 & 0.0222 \\
QV-mt & 0.0827 & 0.0605 & 0.0468 \\
QQ-mt & 0.0611 & 0.0622 & 0.0647 \\
VQ-mt & 0.0232 & 0.0232 & 0.0232 \\
\hline VV-hm & 0.0064 & 0.0064 & 0.0064 \\
QV-hm & 0.0066 & 0.0066 & 0.0066 \\
QQ-hm & 0.0262 & 0.0262 & 0.0262 \\
VQ-hm & 0.0222 & 0.0213 & 0.0208 \\
\hline
\end{tabular}

According to the Table 2, we can know that comparing with selecting Q-PV to access in the middle and tail of the feeder, selecting V-PV has a significant effect on suppressing the voltage fluctuation. Selecting Q-PV to access in the head of the feeder has a similar effect as selecting V-PV. In conclusion, it is advised to select Q-PV to access in the head and V-PV to access in the middle and tail of the feeder.

\section{Conclusion}

In this paper, a multi-time scale coordinated control method based on PCC reactive power/voltage optimization of photovoltaic is proposed, including an ORPD model on the long-time scale and a Q/VASOC strategy on the short-time scale. By comparing the control effect of Q-PV and V-PV accessed from different locations in DN, an advise on the coordinated control method for multiple PVs is provided. In conclusion, it is advised that select Q-PV to access in the head and V-PV to access in the middle and tail of the feeder, which has a significant effect on reducing power loss, action times of discrete devices and suppressing the voltage fluctuation.

\section{Acknowledgment}

This work was supported by the National Natural Science Foundation of China (51377060).

\section{References}

[1] Haque MM, Wolfs P. A review of high PV penetrations in LV distribution networks: present status, impacts and mitigation measures. Renewable and Sustainable Energy Reviews, 2016; 62(2): 1195-1208.

[2] Chen X, Zhang YJ, Huang XM. Review of reactive power and voltage control method in the background of active distribution network. Automation of Electric Power System, 2016; 40(1): 143-151.

[3] Calderaro V, Conio G, Galdi V, Massa G, Piccolo A. Optimal decentralized voltage control for distribution systems with 
inverter -based distributed generators. IEEE Transactions on Power System, 2013; 29(1): 230-241.

[4] Ren JY, Gu W, Wang Y, Ji WL, Liu HB, Cao G. Multi-time scale active and reactive power coordinated op-timal dispatch in active distribution network based on model predictive control. Proceedings of the CSEE, 2017; 37(12): 1-11.

[5] Mayhorn E, Xie L, Butler-Purry K. Multi-time scale coordination of distributed energy resources in isolated power systems. IEEE Transactions on Power System, 2017; 8(2): 998-1005.

[6] Tang Y, Liu YQ, Ning J, Zhao JB. Multi-time scale coordinated scheduling strategy with distributed power flow controllers for minimizing wind power spillage. Energies, 2017; 10(11): 1804.

[7] Yu WP, Liu D, Yu NH. Feeder control error and its application in coordinate control of active distribution network. Proceedings of the CSEE, 2013; 33(13): 108-115.

[8] Huang W, Liu SL, Wang W, Yi YQ, Zhang YJ. Optimal reactive power dispatch with long-scale in distribu-tion network considering uncertainty of photovoltaic. Automation of Electric Power Systems, 2018; 42(5): 154-162. 